\title{
Supplementary Data for The Dual Alignments of the Solstitial Churches in North Wales
}

\section{Bernadette Brady}

University of Wales Trinity Saint David

b.brady@tsd.ac.uk

TABLE SF 1. The declination of Welsh historical churches - North Wales subset

Data is sorted from highest to lowest value for Dec E. The churches that have eastern declinations that are judged to be orientated towards the summer solstice are emphasised with a grey band.

Key: Alt E = altitude east; Alt W = altitude west; Az E = true azimuth east; Dec E = declination east; Dec W = declination west; HWT = Live link to HeyWhatsThat image for the churches'locations; $L F=$ live field work; $V F=$ virtual field work

\begin{tabular}{|c|c|c|c|c|c|c|c|c|c|c|c|c|}
\hline ID & Method & Name & Town & Historic County & Longitude & Latitude & AzE & Alt E & Dec $E$ & Alt $W$ & Dec W & HWT \\
\hline HC169 & VF & St Stephen's Church & Bodfari & Flintshire & -3.3606 & 53.2203 & 32.5 & 11.5 & 40.88304218 & 1.5 & -28.93602741 & http://www.heywhatsthat.com/main-0904.html?view=IRIP5SJV \\
\hline HC176 & VF & St Michael's Church & Abergele & Denbighshire & -3.583417 & 53.282944 & 7.5 & 0 & 36.35232838 & 4 & -32.37025911 & http://www.heywhatsthat.com/main-0904.html?view=MQVG9N4E \\
\hline HC141 & LF & $\begin{array}{l}\text { St Cynfarch and St Mary's } \\
\text { Church }\end{array}$ & Llanfair Dyffryn & Denbighshire & -3.293755 & 53.089522 & 38 & 3.5 & 31.41163774 & 2.6 & -25.88043212 & http://www.heywhatsthat.com/main-0904.html?view=EUT506KB \\
\hline HC142 & VF & St Mary's Church & Betws-y-Coed & Caernarfonshire (east) & -3.802839 & 53.091554 & 42 & 3.5 & 29.62152885 & 23.7 & -5.00554893 & http://www.heywhatsthat.com/main-0904.html?view=RJJ8VNAH \\
\hline HC146 & VF & St Mary's Church & Cyffylliog & Denbighshire & -3.407209 & 53.109345 & 58 & 10.5 & 27.29211255 & 3.2 & -15.84072179 & http://www.heywhatsthat.com/main-0904.html?view=087BC43Q \\
\hline HC148 & VF & St Mwrog's Church & | llanfwrog & Denbighshire & -3.325609 & 53.110242 & 48.5 & 3.6 & 26.5635541 & 4.5 & -19.49828528 & http://www.heywhatsthat.com/main-0904.html?view=IPSOI7HN \\
\hline HC157 & LF & St Cynhafal's Church & \begin{tabular}{|l} 
Llangynhafal, \\
Denbigh
\end{tabular} & Denbighshire & -3.297999 & 53.160658 & 61 & 11.5 & 26.38505624 & 1.4 & -15.72607685 & http://www.heywhatsthat.com/main-0904.html?view=CNOIL224 \\
\hline HC127 & LF & St Trillo's Church & \begin{tabular}{|l} 
Llandrillo yn \\
Edeyrnion
\end{tabular} & Denbighshire & -3.437787 & 52.922365 & 54 & 4.5 & 24.57458921 & 7 & -14.74411215 & http://www.heywhatsthat.com/main-0904.html?view=PEF36K52 \\
\hline HC177 & LF & $\begin{array}{l}\text { St Mael and St Sulien's } \\
\text { Church }\end{array}$ & Cwm & Flintshire & -3.4378 & 53.285896 & 65.5 & 12.5 & 24.55336443 & 0.4 & -14.02294995 & http://www.heywhatsthat.com/main-0904.html?view=V8MJDEL0 \\
\hline HC190 & LF & St Dwynwen's Church & Llanddwyn & Anglesey & -4.412903 & 53.1375 & 48 & 0.7 & 24.27733756 & 0 & -23.66630683 & http://www.heywhatsthat.com/main-0904.html?view=IDI9BVOJ \\
\hline HC165 & LF & St Cwyfan's Church & Llandyrnog & Denbighshire & -3.317818 & 53.186399 & 63 & 10.3 & 24.25513416 & 1.3 & -14.70273144 & http://www.heywhatsthat.com/main-0904.html?view=N9AJKVCT \\
\hline
\end{tabular}


Table SF1 Continued

\begin{tabular}{|c|c|c|c|c|c|c|c|c|c|c|c|c|}
\hline ID & Method & Name & Town & Historic County & Longitude & Latitude & AzE & Alt $\mathrm{E}$ & Dec E & Alt $\mathrm{W}$ & DecW & HWT \\
\hline HC153 & LF & St Rhychwyn's Church & Llanrhychwyn & Caernarfonshire (east) & -3.832992 & 53.137542 & 49 & 1 & 24.04604103 & 6.5 & -17.48559237 & http://www.heywhatsthat.com/main-0904.html?view=U2NJPJ4P \\
\hline HC233 & LF & $\begin{array}{l}\text { St Michael and All Angels } \\
\text { Church }\end{array}$ & Llanrug & Caernarfonshire (central) & -4.2039523 & 53.144478 & 50 & 1.5 & 23.97618638 & 0 & -22.67748754 & http://www.heywhatsthat.com/main-0904.html?view=K7DQGPI6 \\
\hline HC185 & LF & St Beuno's Church & Aberffraw & Anglesey & -4.4662495 & 53.190751 & 48 & 0.3 & 23.89727523 & 1.5 & -22.32228294 & http://www.heywhatsthat.com/main-0904.html?view=C6C16C7Q \\
\hline HC191 & LF & St Eugrad's Church & Llaneugrad & Anglesey & -4.260823 & 53.332958 & 48 & -0.2 & 23.37692541 & 4.4 & -19.68599562 & http://www.heywhatsthat.com/main-0904.html?view=A4K905SP \\
\hline HC145 & $\mathbf{L F}$ & St Garmon's Church & Llanarmon-yn-lal & Denbighshire & -3.2099 & 53.0965 & 58 & 5 & 22.74839525 & 2.5 & -16.44049925 & http://www.heywhatsthat.com/main-0904.html?view=M8B324SC \\
\hline HC225 & LF & St Deiniol's Cathedral & Bangor & Caernarfonshire (central) & -4.127501 & 53.22652 & 55 & 3 & 22.63282485 & 2.3 & -18.11653154 & http://www.heywhatsthat.com/main-0904.html?view=M8SOHS53 \\
\hline HC159 & LF & St Tyrnog's Church & $\begin{array}{l}\text { Llandyrnog, } \\
\text { Denbigh }\end{array}$ & Denbighshire & -3.3363 & 53.1755 & 58.5 & 5 & 22.44 & 1.7 & -16.81503 & http://www.heywhatsthat.com/main-0904.html?view=UFMON4MF \\
\hline HC188 & LF & St Peulan's Church & Llanbeulan & Anglesey & -4.440916 & 53.251337 & 54 & 2 & 22.29 & 0.6 & -20.07595 & http://www.heywhatsthat.com/main-0904.html?view=T1E1EV50 \\
\hline HC154 & LF & St Hychan's Church & Llanychan & Denbighshire & -3.325644 & 53.1487 & 61 & 5 & 21.0630456 & 2.6 & -14.72389542 & http://www.heywhatsthat.com/main-0904.html?view=EBCGOBAQ \\
\hline M226 & VF & St Mary's Priory & Beddgelert & Caernarfonshire (central) & -4.101741 & 53.010753 & 67 & 9 & 20.92507 & 14.7 & -1.415666686 & http://www.heywhatsthat.com/main-0904.html?view=G782VQDO \\
\hline HC246 & $\mathrm{LF}$ & Old St Peter's Church & $\begin{array}{l}\text { Llandedr Dyffryn } \\
\text { Clywd }\end{array}$ & Denbighshire & -3.2792631 & 53.128482 & 67 & 9 & 20.89803 & 1 & -12.73547563 & http://www.heywhatsthat.com/main-0904.html?view=ILPVC92V \\
\hline HC197 & $\mathrm{LF}$ & St Cwyfan's Church & Llangwyfan & Anglesey & -4.491992 & 53.185534 & 56 & 1.3 & 20.68069054 & 0 & -19.57757217 & http://www.heywhatsthat.com/main-0904.html?view=06CLIDUV \\
\hline HC129 & $\mathrm{LF}$ & Llangar Church & Llangar & Denbighshire & -3.3959 & 52.9712 & 69 & 9.6 & 20.23856228 & 2.6 & -10.33353907 & http://www.heywhatsthat.com/main-0904.html?view=MOABJO46 \\
\hline HC243 & VF & St Beuno's Church & Pistyll & Caernarfonshire (west) & -4.4897832 & 52.95215 & 72 & 11.8 & 20.20973639 & -0.2 & -10.89221269 & http://www.heywhatsthat.com/main-0904.html?view=JQB59FDM \\
\hline HC180 & $\mathrm{VF}$ & St Melyd's Church & Meliden & Flintshire & -3.408148 & 53.318459 & 72 & 10.5 & 19.1263533 & 1 & -9.821084198 & http://www.heywhatsthat.com/main-0904.html?view=IJ52V5PM \\
\hline HC170 & VF & Church of Corpus Christi & Tremeirchion & Flintshire & -3.376 & 53.246798 & 67 & 6.3 & 18.68173208 & 0.8 & -12.86131757 & http://www.heywhatsthat.com/main-0904.html?view=D5LO8IPI \\
\hline HC245 & $\mathrm{VF}$ & St Beuno's Church & Gwyddel Wern & Denbighshire & -3.380495 & 53.009451 & 68 & 5.8 & 17.75562757 & 6.2 & -7.921333487 & http://www.heywhatsthat.com/main-0904.html?view=E8SBED7T \\
\hline HC163 & LF & St Marcella's Church & Llanfarchell & Denbighshire & -3.3898 & 53.1848 & 65.5 & 3.5 & 17.27206861 & 2.8 & -12.06930281 & http://www.heywhatsthat.com/main-0904.html?view=04U3U6LO \\
\hline HC138 & LF & $\begin{array}{l}\text { St Trillo's Church } \\
\text { (Foddhyd?) }\end{array}$ & Clocaenog & Denbighshire & -3.371907 & 53.077371 & 64 & 2.3 & 17.17051668 & 5.5 & -10.69082728 & http://www.heywhatsthat.com/main-0904.html?view=H2J1PIGQ \\
\hline HC155 & LF & $\begin{array}{l}\text { St Saeran's Church (6th } \\
\text { cent. monastery) }\end{array}$ & $\begin{array}{l}\text { Llanynys, } \\
\text { Denbighshire }\end{array}$ & Denbighshire & -3.342531 & 53.153607 & 67.5 & 4.7 & 17.11459291 & 2.6 & -11.12479792 & http://www.heywhatsthat.com/main-0904.html?view=HPH52IHK \\
\hline HC196 & VF & St Christiolus' Church & Llangristiolus & Anglesey & -4.3237625 & 53.236495 & 63 & 1 & 16.59826688 & 1 & -14.93343555 & http://www.heywhatsthat.com/main-0904.html?view=NC7AMTT6 \\
\hline HC178 & VF & $\begin{array}{l}\text { St Bridget's (Ffaid or Bride) } \\
\text { Church }\end{array}$ & Dyserth & Denbighshire & -3.417821 & 53.303047 & 74 & 8.7 & 16.50523025 & 1.5 & -8.260312647 & http://www.heywhatsthat.com/main-0904.html?view=TN747182 \\
\hline HC167 & VF & St Cadwaladr's Church & Llangadwaladr & Anglesey & -4.430968 & 53.195872 & 63 & 0.5 & 16.19779152 & 0 & -15.78195648 & http://www.heywhatsthat.com/main-0904.html?view=DJ2NDA5F \\
\hline HC199 & VF & St lestyn's Church & Llaniestyn & Anglesey & -4.1244726 & 53.294409 & 62 & -0.3 & 16.04564911 & 1.4 & -15.12544671 & http://www.heywhatsthat.com/main-0904.html?view=U1 1UGJLP \\
\hline
\end{tabular}




\begin{tabular}{|c|c|c|c|c|c|c|c|c|c|c|c|c|}
\hline ID & Method & Name & Town & Historic County & Longitude & Latitude & AzE & Alt E & Dec E & Alt $W$ & DecW & HWT \\
\hline HC182 & VF & Hen Capel & Lligwy & Anglesey & -4.256489 & 53.352442 & 63.5 & 0 & 15.44608446 & 2 & -13.77847432 & http://www.heywhatsthat.com/main-0904.html?view=JSNGV96Q \\
\hline HC174 & VF & St Winefride's Chapel & Holywell & Flintshire & -3.2232277 & 53.277176 & 72 & 5.6 & 15.19526971 & 8.8 & -3.438436408 & http://www.heywhatsthat.com/main-0904.html?view=A8L5DEKC \\
\hline HC239 & VF & St Maelrhys' Church & Llanfaelrhys & Caernarfonshire (west) & -4.6570028 & 52.809343 & 70 & 4 & 15.17731222 & -0.1 & -12.01282968 & http://www.heywhatsthat.com/main-0904.html?view=VCQ7BVNE \\
\hline HC230 & VF & St Mary's Church & $\begin{array}{l}\text { Lanfair-is-Gaer (Bryn } \\
\text { Llanfair) }\end{array}$ & Caernarfonshire (central) & -4.24304 & 53.16997353 & 68 & 2.55 & 15.06677061 & 0.2 & -12.81242789 & http://www.heywhatsthat.com/main-0904.html?view=VTS8SVAK \\
\hline HC184 & VF & St Patrick's Church & Llanbadrig & Anglesey & -4.445423 & 53.423465 & 69 & 2.6 & 14.46327989 & 0 & -12.33047531 & http://www.heywhatsthat.com/main-0904.html?view=SL4K8TBC \\
\hline HC132 & VF & St Mary's Church & Pentraeth & Anglesey & -4.214684 & 53.283327 & 71.5 & 4 & 14.19117516 & 0.7 & -10.36369865 & http://www.heywhatsthat.com/main-0904.html?view=A3P45IHU \\
\hline HC143 & VF & St Michael's Church & Efenechtyd & Denbighshire & -3.328128 & 53.091795 & 76 & 6.7 & 13.74375878 & 7.2 & -2.517275891 & http://www.heywhatsthat.com/main-0904.html?view=JDKOVTDD \\
\hline HC198 & VF & St Nidan's Church & Llanidan & Anglesey & -4.253469 & 53.177858 & 68.5 & 0.9 & 13.42679956 & 0 & -12.68884539 & http://www.heywhatsthat.com/main-0904.html?view=AFEQAFLI \\
\hline HC123 & VF & St Hywyn's Church & Aberdaron & Caernarfonshire (west) & -4.711426 & 52.80383 & 73 & 4 & 13.40822135 & 3 & -7.748246393 & http://www.heywhatsthat.com/main-0904.html?view=ARBF1806 \\
\hline HC228 & VF & St Peblig's Church & Llanbeblig & Caernarfonshire (central) & -4.262475 & 53.136216 & 71 & 2.5 & 13.2985735 & 0.1 & -11.18143131 & http://www.heywhatsthat.com/main-0904.html?view=DLLL5V3C \\
\hline HC205 & VF & St Ceidio's Church & Rhodwydd Geidio & Anglesey & -4.3876165 & 53.342289 & 68 & 0.1 & 13.00595753 & 1.5 & -11.68765787 & http://www.heywhatsthat.com/main-0904.html?view=GG80E9BJ \\
\hline HC130 & LF & $\begin{array}{l}\text { St Mael and St Sulien's } \\
\text { Church }\end{array}$ & Corwen & Denbighshire & -3.373259 & 52.980096 & 73 & 3.2 & 12.72839695 & 10.1 & -1.907693776 & http://www.heywhatsthat.com/main-0904.html?view=G8VVMNEU \\
\hline HC195 & VF & St Ceinwen's Church & Llangeinwen & Anglesey & -4.3354784 & 53.16658 & 69.5 & 0.7 & 12.69191909 & 1 & -11.29995957 & http://www.heywhatsthat.com/main-0904.html?view=DOQSD264 \\
\hline M175 & LF & $\begin{array}{l}\text { St Mary and All Saints } \\
\text { Church }\end{array}$ & Conwy & Denbighshire & -3.828826 & 53.280639 & 70 & 1 & 12.61805346 & 4.2 & -8.351033222 & http://www.heywhatsthat.com/main-0904.html?view=J195F5NA \\
\hline HC131 & LF & St Tysilio's Church & Llantysilio & Denbighshire & -3.201852 & 52.983232 & 79 & 7.5 & 12.59824732 & 3.4 & -3.860093166 & http://www.heywhatsthat.com/main-0904.html?view=VASCDO4R \\
\hline HC248 & VF & St Eilian's Church & Llaneilian-yn-Rhos & Denbighshire & -3.7056662 & 53.272642 & 70 & 0.8 & 12.45667821 & 4.4 & -8.188970711 & http://www.heywhatsthat.com/main-0904.html?view=F2ACVBND \\
\hline HC237 & VF & St Pedrog's Church & Llanbedrog & Caernarfonshire (west) & -4.482566 & 52.855487 & 70 & 0.6 & 12.40714048 & 7.6 & -5.697907735 & http://www.heywhatsthat.com/main-0904.html?view=G6IBN8JG \\
\hline HC156 & $\mathrm{LF}$ & St Dyfnog's Church & Denbigh (LL16 4NN) & Denbighshire & -3.376275 & 53.16042 & 74 & 3.3 & 12.18454612 & 5.4 & -5.11849057 & http://www.heywhatsthat.com/main-0904.html?view=N3BNLRI4 \\
\hline HC149 & $\mathrm{LF}$ & St Peter's Church & Ruthin & Denbighshire & -3.3108 & 53.1154 & 75 & 4 & 12.16692783 & 2.5 & -6.909873075 & http://www.heywhatsthat.com/main-0904.html?view=U7VB776N \\
\hline HC187 & VF & St B(P)abo's Church & Llanbabo & Anglesey & -4.4383384 & 53.352913 & 71 & 0.2 & 11.36894711 & 2.8 & -8.911058635 & http://www.heywhatsthat.com/main-0904.html?view=JR3S5RQF \\
\hline HC133 & VF & St Beuno's Church & Clynnog Fawr & Caernarfonshire (central) & -4.365325 & 53.020999 & 78.5 & 5.5 & 11.29958865 & 0 & -6.887731128 & http://www.heywhatsthat.com/main-0904.html?view=N91A6UH2 \\
\hline HC172 & VF & $\begin{array}{l}\text { St Mary and St Nicholas' } \\
\text { Church }\end{array}$ & Beaumaris & Anglesey & -4.094462 & 53.263465 & 71 & 0 & 11.22919237 & 4 & -7.952802312 & http://www.heywhatsthat.com/main-0904.html?view=PFEPQA73 \\
\hline HC229 & VF & St Baglan's Church & Llanfaglan & Caernarfonshire (central) & -4.309516 & 53.12102653 & 75.5 & 2.7 & 10.82288277 & 0 & -8.641978547 & http://www.heywhatsthat.com/main-0904.html?view=BSOVP9AJ \\
\hline HC150 & VF & St Mary's Church & $\begin{array}{l}\text { Llanfair Mathafarn } \\
\text { Eithaf }\end{array}$ & Anglesey & -4.243501 & 53.321835 & 72 & 0 & 10.63675682 & 2 & -9.002637882 & http://www.heywhatsthat.com/main-0904.html?view=15N3IC4V \\
\hline HC136 & VF & St Elidan's Church & Llanelidan & Denbighshire & -3.329147 & 53.044696 & 78 & 3.8 & 10.23464337 & 4.6 & -3.468790967 & http://www.heywhatsthat.com/main-0904.html?view=RJ5J59OI \\
\hline
\end{tabular}




\begin{tabular}{|c|c|c|c|c|c|c|c|c|c|c|c|c|}
\hline ID & Method & Name & Town & Historic County & Longitude & Latitude & AzE & Alt E & Dec E & Alt $\mathbf{w}$ & DecW & HWT \\
\hline HC160 & VF & St Mary's Church & Cilcain & Flintshire & -3.2325 & 53.1769 & 73 & 0 & 10.09215489 & 4.2 & -6.669088918 & http://www.heywhatsthat.com/main-0904.html?view=TTOPUKOO \\
\hline HC192 & VF & St Mary's Church & Llanfair-yn-Neubwll & Anglesey & -4.5551647 & 53.270048 & 75.5 & 1.3 & 9.664745055 & 1.2 & -7.638412314 & http://www.heywhatsthat.com/main-0904.html?view=V4U320KJ \\
\hline HC242 & VF & St Mary's Church & Nefyn & Caernarfonshire (west) & -4.518043 & 52.936499 & 83 & 6.7 & 9.558150802 & 1.5 & -3.011539948 & http://www.heywhatsthat.com/main-0904.html?view=CNPQ1BS7 \\
\hline HC144 & VF & St Michael's Church & Betws-y-Coed & Caernarfonshire (east) & -3.799444 & 53.0925 & 92 & 12 & 8.380562636 & 8.5 & 7.985189005 & http://www.heywhatsthat.com/main-0904.html?view=TQKQLJJ6 \\
\hline HC183 & VF & St Eilian's Church & Llaneilian & Anglesey & -4.30477 & 53.409737 & 76 & 0 & 8.291351973 & 0.5 & -7.885545279 & http://www.heywhatsthat.com/main-0904.html?view=EHIEP3DR \\
\hline HC221 & VF & St Mary's Church & Caerhun & Caernarfonshire (east) & -3.833306 & 53.216518 & 86.5 & 7.7 & 8.252504702 & 5.7 & 2.47428004 & http://www.heywhatsthat.com/main-0904.html?view=Q84K1DEQ \\
\hline HC168 & VF & St Michael's Church & Nannerch & Flintshire & -3.249452 & 53.217569 & 82 & 2.8 & 7.028256481 & 3 & -2.367157323 & http://www.heywhatsthat.com/main-0904.html?view=029E2M21 \\
\hline HC128 & VF & St Collen's Church & Llangollen & Denbighshire & -3.167739 & 52.969417 & 82 & 1.5 & 6.008988775 & 8.6 & 2.091920889 & http://www.heywhatsthat.com/main-0904.html?view=C57K8A3J \\
\hline HC224 & VF & St Celynin's Church & Llangelynnin & Caernarfonshire (east) & -3.872981 & 53.245823 & 81 & 0.2 & 5.532128365 & 6.5 & -0.132149045 & http://www.heywhatsthat.com/main-0904.html?view=ANHMPEU1 \\
\hline HC204 & LF & St Beuno's Church & Tredfraeth & Anglesey & -4.3842678 & 53.206805 & 82 & 0.6 & 5.263485554 & 0.8 & -4.138385169 & http://www.heywhatsthat.com/main-0904.html?view=TAO37DQT \\
\hline HC137 & VF & St Mary's Church & Derwen & Denbighshire & -3.388681 & 53.045449 & 82 & 0.5 & 5.200344534 & 5 & -0.7851275 & http://www.heywhatsthat.com/main-0904.html?view=TOQU2CRD \\
\hline HC200 & LF & St Rhwydrus' Church & Llanrhwydrus & Anglesey & -4.5257476 & 53.4091 & 82 & 0.3 & 5.000451901 & 0 & -4.758768382 & http://www.heywhatsthat.com/main-0904.html?view=GDE310RR \\
\hline HC152 & $\mathrm{VF}$ & St Berres' Church & llanferres & Denbighshire & -3.214488 & 53.135984 & 89 & 5.5 & 4.997042083 & 10 & 7.389755834 & http://www.heywhatsthat.com/main-0904.html?view=KJ45R5TM \\
\hline HC234 & VF & St Beuno's Church & Penmorpha & Caernarfonshire (central) & -4.1720702 & 52.940177 & 86.5 & 2.5 & 4.103843922 & 5.2 & 2.045091965 & http://www.heywhatsthat.com/main-0904.html?view=DFQ08HG4 \\
\hline HC194 & VF & St Figael's Church & Llanfigael & Anglesey & -4.5114259 & 53.315842 & 84.5 & 0.7 & 3.844659442 & 0.3 & -3.041477184 & http://www.heywhatsthat.com/main-0904.html?view=FPBI4364 \\
\hline HC241 & VF & Holy Cross Church & Llanor & Caernarfonshire (west) & -4.449205 & 52.907517 & 85 & 0.96 & 3.77972962 & 1 & -2.214160557 & http://www.heywhatsthat.com/main-0904.html?view=LP1BIAOB \\
\hline HC227 & VF & St Aelhaearn's Church & Llanaelhaearn & Caernarfonshire (central) & -4.403704 & 52.976342 & 90.5 & 5 & 3.689496314 & 13.9 & 11.35548623 & http://www.heywhatsthat.com/main-0904.html?view=VI1AAH6T \\
\hline HC240 & $\mathrm{VF}$ & St Gwyn's Church & Llangynnadl & Caernarfonshire (west) & -4.6623994 & 52.866716 & 90 & 4.3 & 3.426929257 & 1.6 & 1.275512984 & http://www.heywhatsthat.com/main-0904.html?view=BNLOLDIN \\
\hline HC201 & VF & St Sadwrn's Church & Llansadwrn & Anglesey & -4.169269 & 53.259842 & 85 & 0.2 & 3.148977869 & 1.4 & -1.864789956 & http://www.heywhatsthat.com/main-0904.html?view=S4V3FFCV \\
\hline HC181 & $\mathrm{VF}$ & St Gallgo's Church & Llanallgo & Anglesey & -4.252399 & 53.341152 & 85 & 0 & 2.982805248 & 1.5 & -1.77755199 & http://www.heywhatsthat.com/main-0904.html?view=QSPD6014 \\
\hline HC231 & VF & St Cybi's Church & Llangybi & Caernarfonshire (central) & -4.340131 & 52.94512552 & 86 & 0.7 & 2.968135956 & 2.3 & -0.571388138 & http://www.heywhatsthat.com/main-0904.html?view=M8PPKVHL \\
\hline HC125 & VF & St Engan's Church & Llanengan & Caernarfonshire (west) & -4.53282 & 52.81375 & 96 & 8.2 & 2.928917114 & 1.3 & 4.659614778 & http://www.heywhatsthat.com/main-0904.html?view=N3DLQIQ7 \\
\hline HC162 & LF & St Tysilio's Church & Church Island & Anglesey & -4.17126 & 53.22242 & 92 & 4.5 & 2.407863167 & 3 & 3.599703588 & http://www.heywhatsthat.com/main-0904.html?view=DPOFIJPA \\
\hline HC249 & LF & St Cynfran's Church & Llysfaen & Denbighshire & -3.661473 & 53.282824 & 86 & 0 & 2.390212983 & 2.6 & -0.303625766 & http://www.heywhatsthat.com/main-0904.html?view=TG03E15H \\
\hline
\end{tabular}


S5 Bernadette Brady

Table SF1 Continued

\begin{tabular}{|c|c|c|c|c|c|c|c|c|c|c|c|c|}
\hline ID & Method & Name & Town & Historic County & Longitude & Latitude & AzE & Alt E & DecE & Alt $w$ & DecW & HWT \\
\hline HC203 & VF & St Michael's Church & Penrhos Llugwy & Anglesey & -4.28406 & 53.348283 & 88 & 1 & 1.996116338 & 4.4 & 2.337068749 & http://www.heywhatsthat.com/main-0904.html?view=PJSO8QB9 \\
\hline HC238 & VF & St Tudwen's Church & Llandudwen & Caernarfonshire (west) & -4.5676048 & 52.901423 & 88 & 0.8 & 1.844390732 & 0.7 & -0.64774982 & http://www.heywhatsthat.com/main-0904.html?view=PCE3TIV1 \\
\hline HC186 & VF & St Llwddian's Church & Heneglwys & Anglesey & -4.3664928 & 53.258633 & 88 & 0.6 & 1.677136272 & 0.7 & -0.635161706 & http://www.heywhatsthat.com/main-0904.html?view=D30AE0F4 \\
\hline HC250 & VF & St Mary's Church & Rhuddlan & Flintshire & -3.4694736 & 53.290792 & 90.5 & 2.4 & 1.625075249 & 0.5 & 0.699713922 & http://www.heywhatsthat.com/main-0904.html?view=JCQ4485C \\
\hline HC254 & VF & $\begin{array}{l}\text { St Kentigern and St Asa's } \\
\text { Church }\end{array}$ & St Asaph & Denbighshire & -3.4452613 & 53.257016 & 94 & 3.8 & 0.657119414 & 1.3 & 3.434038624 & http://www.heywhatsthat.com/main-0904.html?view=H3D2MLK5 \\
\hline HC202 & VF & St Cynfarwy's Church & Llechcynfarwy & Anglesey & -4.430737 & 53.301976 & 89 & 0 & 0.59757799 & -0.1 & -0.677761637 & http://www.heywhatsthat.com/main-0904.html?view=CQ0C25IQ \\
\hline HC164 & VF & St Mary's Church & $\begin{array}{l}\text { Llanfair-yng- } \\
\text { Nghornwy }\end{array}$ & Anglesey & -4.516384 & 53.38793 & 90 & 0.5 & 0.401344116 & 4.8 & 3.851315172 & http://www.heywhatsthat.com/main-0904.html?view=E9122HE2 \\
\hline HC252 & VF & $\begin{array}{l}\text { St Mary Magadalene's } \\
\text { Church }\end{array}$ & Gwaenysgor & Flintshire & -3.3896832 & 53.318073 & 90 & 0.1 & 0.080196397 & 0.03 & 0.024058923 & http://www.heywhatsthat.com/main-0904.html?view=M7MJI9U8 \\
\hline HC193 & VF & St Mechell's Church & Llanfechell & Anglesey & -4.453619 & 53.393029 & 96.4 & 0.9 & -3.087115734 & 1.8 & 5.258736893 & http://www.heywhatsthat.com/main-0904.html?view=SE2O5Q1A \\
\hline HC179 & LF & St Cybi's Church & Holyhead & Anglesey & -4.632515 & 53.31131 & 96 & 0 & -3.580582968 & 3.7 & 6.549996445 & http://www.heywhatsthat.com/main-0904.html?view=JK2CSA2M \\
\hline HC134 & LF & St Michael's Church & $\begin{array}{l}\text { Llanfihangel Glyn } \\
\text { Myfyr }\end{array}$ & Denbighshire & -3.5081 & 53.0325 & 106 & 7 & -3.85039517 & 4.5 & 13.17545787 & http://www.heywhatsthat.com/main-0904.html?view=L9KONDFH \\
\hline HC236 & VF & St Cawrdaf's Church & Abererch & Caernarfonshire (west) & -4.3854506 & 52.902667 & 98 & 1 & -4.014683638 & 2.7 & 6.97432974 & http://www.heywhatsthat.com/main-0904.html?view=A7TKIJLV \\
\hline HC158 & VF & St Mary's Church & Mold & Flintshire & -3.142947 & 53.169131 & 99 & 0.8 & -4.737501827 & 2.1 & 7.067718903 & http://www.heywhatsthat.com/main-0904.html?view=MVM8RNMI \\
\hline HC189 & VF & St Mary's Church & Llanbeulan & Anglesey & -4.4483323 & 53.227721 & 107 & 1.7 & -8.695543797 & 0.1 & 10.16142268 & http://www.heywhatsthat.com/main-0904.html?view=TAUDOVOV \\
\hline HC223 & VF & St Tudno's Church & Llandudno & Caernarfonshire (east) & -3.8491376 & 53.337039 & 110 & 2 & -10.14285652 & 4 & 15.05113626 & http://www.heywhatsthat.com/main-0904.html?view=NFO55377 \\
\hline HC244 & VF & St Aelrhiw's Church & $\begin{array}{l}\text { Rhiw with } \\
\text { Llanfaelrhys }\end{array}$ & Caernarfonshire (west) & -4.6225257 & 52.826193 & 108.5 & 0.6 & -10.56623454 & 11.5 & 20.28775468 & http://www.heywhatsthat.com/main-0904.html?view=NS4KT5NR \\
\hline HC247 & LF & St Trillo's Chapel & Llandrillo-yn-Rhos & Denbighshire & -3.7396847 & 53.314413 & 114 & 1.2 & -13.07030217 & 2 & 15.71382874 & http://www.heywhatsthat.com/main-0904.html?view=NOLDR7GF \\
\hline
\end{tabular}

